\title{
Measuring mammographic density: comparing a fully automated volumetric assessment versus European radiologists' qualitative classification
}

\author{
Hanna Sartor ${ }^{1,2}$ - Kristina Lång ${ }^{1,2}$ • Aldana Rosso ${ }^{3}$ - Signe Borgquist ${ }^{4,5}$. \\ Sophia Zackrisson ${ }^{1,2} \cdot$ Pontus Timberg $^{6}$
}

Received: 16 December 2015 /Revised: 17 February 2016 / Accepted: 23 February 2016 / Published online: 24 March 2016

(C) The Author(s) 2016. This article is published with open access at Springerlink.com

\begin{abstract}
Objectives Breast Imaging-Reporting and Data System (BI-RADS) mammographic density categories are associated with considerable interobserver variability. Automated methods of measuring volumetric breast density may reduce variability and be valuable in risk and mammographic screening stratification. Our objective was to assess agreement of mammographic density by a volumetric method with the radiologists' classification.

Methods Eight thousand seven hundred and eighty-two examinations from the Malmö Breast Tomosynthesis Screening Trial were classified according to BI-RADS, 4th Edition. Volumetric breast density was assessed using automated software for 8433 examinations. Agreement between volumetric breast density and BI-RADS was
\end{abstract}

Hanna Sartor

hanna.sartor@med.lu.se

1 Medical Radiology, Department of Translational Medicine, Lund University, Lund, Sweden

2 Department of Medical Imaging and Physiology, Skåne University Hospital, Inga Marie Nilssons gata 49, SE-205 02 Malmö, Sweden

3 Epidemiology and Register Centre South (ERC Syd), Skåne University Hospital, Klinkgatan 22, SE-221 85 Lund, Sweden

4 Division of Oncology and Pathology, Department of Clinical Sciences Lund, Lund University, Lund, Sweden

5 Department of Oncology, Skåne University Hospital, Getingevägen 4, SE-221 85 Lund, Sweden

6 Department of Medical Radiation Physics, Department of Translational Medicine, Lund University, Lund, Sweden descriptively analyzed. Agreement between radiologists and between categorical volumetric density and BI-RADS was calculated, rendering kappa values.

Results The observed agreement between BI-RADS scores of different radiologists was $80.9 \%$ [kappa $0.77(0.76-0.79)]$. A spread of volumetric breast density for each BI-RADS category was seen. The observed agreement between categorical volumetric density and BI-RADS scores was $57.1 \%$ [kappa $0.55(0.53-0.56)]$.

Conclusions There was moderate agreement between volumetric density and BI-RADS scores from European radiologists indicating that radiologists evaluate mammographic density differently than software. The automated method may be a robust and valuable tool; however, differences in interpretation between radiologists and software require further investigation.

Key Points

- Agreement between qualitative and software density measurements has not been frequently studied.

- There was substantial agreement between different radiologists' qualitative density assessments.

- There was moderate agreement between software and radiologists' density assessments.

- Differences in interpretation between software and radiologists require further investigation.

Keywords Mammography $\cdot$ Screening $\cdot$ Diagnostic imaging · Breast cancer · Women' health
Abbreviations
BI-RADS Breast Imaging-Reporting and Data System
CC Craniocaudal
DM Digital mammography
DBT Digital breast tomosynthesis 
MBTST Malmö Breast Tomosynthesis Screening Trial

MLO Mediolateral oblique

\section{Introduction}

High mammographic density has consistently been shown to be associated with an increased risk of breast cancer [1]. Hence, there has been a growing interest of evaluating mammographic density for individualized screening programs [2] and for incorporation in risk prediction models [3]. However, optimal use of mammographic density requires a reliable measuring method. Today, both qualitative and quantitative mammographic density measurement methods are available [4]. The most often used clinical classification of mammographic density is the qualitative Breast Imaging-Reporting and Data System (BI-RADS) [5]. Although afflicted with substantial interobserver variability (kappa 0.43-0.79) [6-12], mammographic density as classified by BI-RADS has consistently been associated with an increased risk of breast cancer [1, 13]. However, the latest BI-RADS 5th Edition aims to capture the risk of masking of tumors by dense breast tissue, more than the risk of developing breast cancer [5]. In order to improve objectivity and reproducibility, quantitative breast density measurements have been developed [4]. The areabased, semi-quantitative measurements, such as Cumulus, are represented by different computer-assisted techniques [4]. However, these techniques are also user-dependent and time-consuming. Both the breast itself and the dense breast tissue are three-dimensional, and a previous study reported volumetric breast density measurements to more accurately estimate breast cancer risk than breast density estimated with area-based methods [14]. Previous studies on fully automated volumetric methods of measuring breast density have shown high reproducibility [15] and association with breast cancer risk [16, 17]. Furthermore, the volumetric methods have shown to be positively associated with BI-RADS categories [18-21] as well as to magnetic resonance imaging (MRI) measurements of breast fibroglandular tissue [22, 23]. A previous large study $(n=8867)$ showed good correlation between two different automated techniques of measuring volumetric breast density, but the agreement with visually estimated mammographic density was poor, albeit better than the agreement with the area-based method [24]. In addition to a mere value or a category of mammographic density, temporal changes in mammographic density have also rendered attention. A decrease of mammographic density has been shown to be associated with a decreased risk of contralateral breast cancer [25] as well as a positive marker for response to tamoxifen treatment [26], further motivating a more sensitive measurement than the rather coarse BI-RADS categories.
The aim of this study was to assess the agreement of mammographic density by a fully automated volumetric method with the radiologists' classification according to BI-RADS 4th Edition. Part of the Malmö Breast Tomosynthesis Screening Trial (MBTST) population, comprising nearly 8500 screening mammography examinations with measured volumetric mammographic density and qualitative classification according to BI-RADS, was used to address the aim of this study.

\section{Material and methods}

\section{Malmö breast tomosynthesis screening trial (MBTST)}

The MBTST is a prospective study investigating the use of one-view [mediolateral oblique (MLO)] digital breast tomosynthesis (DBT) alone compared to two-view digital mammography [DM; craniocaudal (CC) and MLO] in a population-based screening program in the city of Malmö, Sweden. The MBTST started in January 2010 and results from the first half of the study population have been described in detail previously [27]. Of 10,547 women invited to the first half of the MBTST, 7500 participated in the study, corresponding to a participation rate of $71.1 \%$ [27]. For all DM (Mammomat Inspiration, Siemens AG, Erlangen, Germany), the anode/filter combination was Wolfram/Rhodium and the automatic exposure control was specified to an average glandular dose of $1.2 \mathrm{mGy}$ (for a 53-mm standard breast consisting of $50 \%$ glandular tissue and $50 \%$ fat tissue) [28]. Raw data from the DM examinations were saved on a dedicated server from February 8, 2012 onwards. This present study was based on the DM examinations with available raw data from February 8, 2012 until March 11, 2014. The study population is illustrated in Fig. 1. The examinations from women with breast cancer with at least 10 months of follow up $(n=100)$ were excluded. Participating women gave written informed consent. This study was approved by the Regional Ethical Review Board at Lund University (Dnr 2009/770) and the local Radiation Safety Board at Skåne University Hospital in Malmö.

\section{BI-RADS composition categories}

A total of 8782 examinations were prospectively classified according to BI-RADS 4th Edition as part of the initial screening reading procedure during the study period. BIRADS 5th Edition was not released at the start of the trial and, hence, it was not used. The following BI-RADS categories for mammographic density were used: BI-RADS 1 , almost fatinvoluted ( $<25 \%$ fibroglandular tissue); BI-RADS 2, scattered fibroglandular densities (25-50\% fibroglandular tissue); BI-RADS 3, heterogeneously dense (51-75\% fibroglandular tissue); and BI-RADS 4, extremely dense 
Fig. 1 Flowchart illustrating study population. *This represents the same examinations. 1 not included in Volpara file, 22 breast implants, 333 missing Volpara values. **This represents the same examinations. Seven examinations without BI-RADS scores

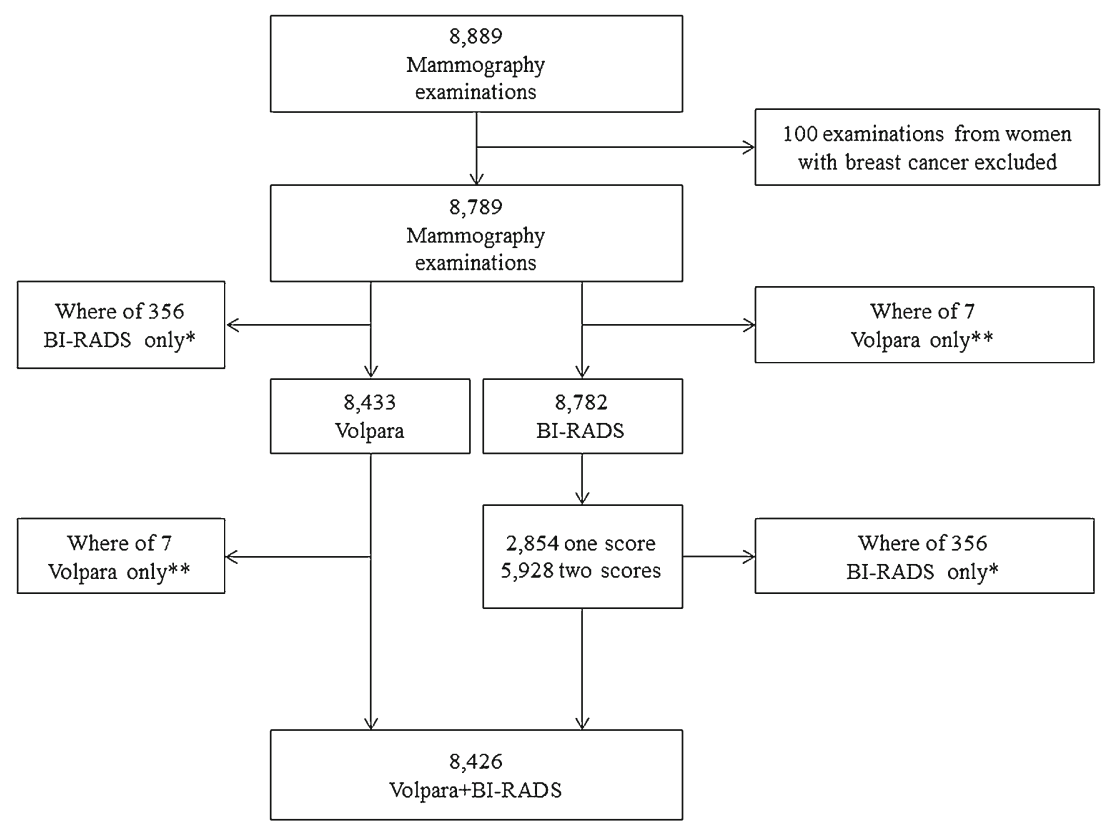

(>75\% fibroglandular tissue). The scores were performed during the ongoing trial by five breast radiologists, all with more than 10 years of experience in breast radiology. Seven examinations were not evaluated with BI-RADS (Volpara only). A total of 2854 examinations had one BI-RADS score. The study protocol was slightly revised to include double reading of mammographic density; a total of 5928 examinations were independently scored according to BI-RADS by different radiologists resulting in two BI-RADS scores for these examinations.

\section{Volumetric breast density analyses by Volpara}

Volpara measures the x-ray attenuation in relevant parts of the breast and relates it to a region in the breast known to only contain adipose tissue (assuming an even breast thickness). Volpara then produces a fibroglandularity content map of the breast that allows for estimation of breast density measurements. The volumetric breast density refers to the percentage of breast density, computed by dividing the fibroglandular tissue volume by the breast volume. A complete description of the method is found elsewhere [29]. Breast density was measured as a continuous variable [volumetric breast density (VBD); ranging from $0 \%$ to approximately $40 \%$ fibroglandular tissue] as well as four ordered categories [Volpara density grade (VDG)]: VDG 1: $<4.5 \%$ fibroglandular tissue, VDG $2: \geq 4.5$ and $<7.5 \%$ fibroglandular tissue, VDG $3: \geq 7.5$ and $<15.5 \%$ fibroglandular tissue, VDG $4: \geq 15.5 \%$ fibroglandular tissue. The thresholds of the VDG categories have been determined by an American expert group of radiologists by recording the average VBD for the assigned BI-RADS category in 500 mammography examinations [29]. The volumetric breast density result was provided per examination by averaging values from the two DM projections (CC and MLO) from both breasts.

A total of 8433 examinations with raw two-view DM data were assessed with a fully automated volumetric breast density measurement software; Volpara (version 1.5.11, Matakina Technology, Wellington, New Zealand). The software calculation was based on four images in $92.4 \%$ of the examinations, but for a few examinations, a lesser amount of images or additional images were assessed (e.g., women with only one breast or software failure for single images). Examinations with previously known breast implants were excluded because the software had known difficulties in correctly measuring volumetric breast density in these images. A total of 356 examinations were not included in analyses with Volpara, predominantly due to lack of DM raw data (Fig. 1). Very few examinations had missing Volpara values due to software failure ( $\leq 5$ cases)

\section{Statistical methods}

Linear-weighted kappa and $95 \%$ confidence interval (CI) values were calculated for estimation of inter-observer variability for examinations with two BI-RADS scores. Agreement between VBD (continuous variable) and BIRADS scores was analyzed descriptively. Kappa values for comparison between VDG (categorical variable with four groups) and BI-RADS scores were calculated using a metaanalysis which means that a separate kappa coefficient was calculated for each reader (reader vs. Volpara). The results were then combined by taking the individual kappa estimates into account and weighting them using the standard error for each kappa, rendering a pooled kappa [30]. By convention, 
values of $<0.0,0.00-0.20,0.21-0.40,0.41-0.60,0.61-0.80$ and $0.81-1.00$ are, respectively, indicative of poor, slight, fair, moderate, substantial and almost perfect agreement [31]. For examinations with two BI-RADS scores, the score from the first radiologist was used. In an additional sensitivity analysis, the score from the second radiologist instead of the score from the first radiologist was used which did not affect the results. In addition, the radiologists were randomly assigned to be reader one or two. For categorical variables, the percentages of cases in which both methods (or both radiologists) agreed were calculated (i.e., observed agreement). Examinations from women with breast cancer $(n=100)$ were excluded in all of the analyses. All the calculations were performed using the software Stata v13 (StataCorp LP, Texas, USA).

\section{Results}

\section{Baseline variables}

The mean age at the study mammography examination was 58 years (range $40-76$ years). Regarding Volpara density values per examination, the median breast volume was $691.1 \mathrm{~cm}^{3}$ (range $40.1-3375.8 \mathrm{~cm}^{3}$ ), the median fibroglandular tissue volume was $49.0 \mathrm{~cm}^{3}$ (range 8.8 $336.6 \mathrm{~cm}^{3}$ ), and the median VBD was $7.2 \%$ (range 1.9$43.3 \%$; Table 1). The examinations with VDG classification were distributed as follows: VDG 1: $20.9 \%$, VDG 2: $32.1 \%$, VDG $3: 31.5 \%$, VDG 4: $15.5 \%$, with a corresponding BIRADS distribution: BI-RADS 1: $16.4 \%$, BI-RADS 2: $40.9 \%$, BI-RADS 3: $35.2 \%$, BI-RADS 4: $7.5 \%$.

\section{Agreement analyses}

There was substantial agreement between BI-RADS scores with a weighted kappa of 0.77 (0.76-0.79; observed agreement $80.9 \%$ ). The distribution of VBD values in relation to BI-RADS categories is shown in Fig. 2. There was a spread of VBD values across each BI-RADS category which might be called poor agreement (Fig. 2). If these two methods of mammographic density measurement were in agreement, we would observe only a certain range of VBD values in each BIRADS category. There was moderate agreement between VDG and BI-RADS, with a pooled kappa for all five radiologists of 0.55 (0.53-0.56; observed agreement $57.1 \%$; Fig. 3). Information regarding which of the categories exhibited the most agreement is shown in Table 2; agreement was highest in BI-RADS 4 and similar for the other groups (BIRADS 1: $60.9 \%$, BI-RADS 2: $50.2 \%$, BI-RADS 3: $57.3 \%$, BI-RADS 4: $85.1 \%$ ). Figures and tables show the crude distribution of human labelling errors without corrections. For a few examinations in the data set $(n=6)$, the BI-RADS scores and VDG values were discrepant over three categories (BIRADS 1 vs. VDG 4). When specifically looking into those examinations, the BI-RADS scores were believed to be labelling errors by the radiologists.

\section{Discussion}

In this large study, we analyzed mammographic density assessment in a screening population with a fully automated volumetric assessment using Volpara software compared to the radiologists' classification according to BI-RADS, 4th Edition. We found that the agreement between BI-RADS scores was substantial, indicating that the radiologists evaluated the mammographic density in a similar manner. Agreement between VDG and BI-RADS scores was moderate.

Our results are in line with a previous large study showing that different mammographic density measurements did not produce identical results [24]. Morrish et al. showed a low

Table 1 Volpara values per BI-RADS category per examination (median, $\min / \max$ )

\begin{tabular}{|c|c|c|c|c|c|c|c|}
\hline BI-RADS $^{\mathrm{a}}$ category $^{\mathrm{b}}$ & $\mathrm{n}$ & $\begin{array}{l}\text { Fibroglandular } \\
\text { tissue volume }\left(\mathrm{cm}^{3}\right)\end{array}$ & $\operatorname{Min} / \max$ & $\begin{array}{l}\text { Breast tissue } \\
\text { volume }\left(\mathrm{cm}^{3}\right)\end{array}$ & $\operatorname{Min} / \max$ & $\begin{array}{l}\text { Volumetric Breast } \\
\text { density }(\%)\end{array}$ & $\operatorname{Min} / \max$ \\
\hline 1 & 1378 & 41.0 & $12.1 / 123.2$ & 1005.7 & $139.1 / 3188.8$ & 4.1 & $1.9 / 26.2$ \\
\hline 2 & 3445 & 44.1 & $11.5 / 184.7$ & 777.5 & $69.6 / 3375.8$ & 5.7 & $2.0 / 32.5$ \\
\hline 3 & 2967 & 60.9 & $8.8 / 257.5$ & 567.0 & $40.1 / 2831.0$ & 10.9 & $2.9 / 32.4$ \\
\hline 4 & 636 & 77.3 & $13.4 / 336.6$ & 360.9 & $56.2 / 1931.2$ & 22.1 & $5.1 / 43.3$ \\
\hline Total $^{\mathrm{c}}$ & 8426 & & & & & & \\
\hline All examinations with Volpara measures ${ }^{\mathrm{d}}$ & 8433 & 49.0 & $8.8 / 336.6$ & 691.1 & $40.1 / 3375.8$ & 7.2 & $1.9 / 43.3$ \\
\hline
\end{tabular}

\footnotetext{
${ }^{a}$ Breast Imaging Reporting and Data System

${ }^{\mathrm{b}}$ BI-RADS score from one reader

${ }^{\mathrm{c}}$ Examinations with BI-RADS score from one reader and Volpara values

${ }^{\mathrm{d}}$ Independent of BI-RADS scores
} 


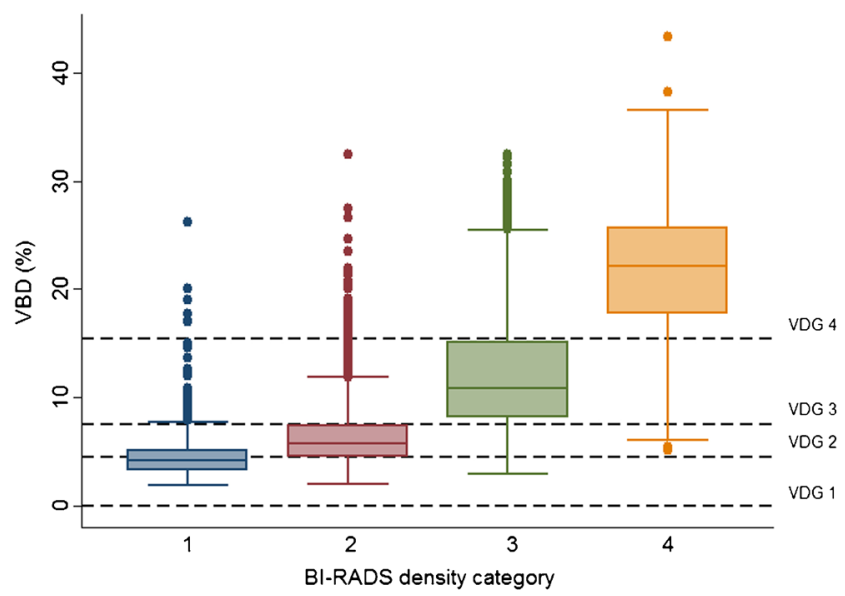

Fig. 2 Box plot showing associations between volumetric breast density (VBD) and BI-RADS

correlation between Volpara and observers' visual estimations of mammographic density using the VAS method (Visual Analog Scale), albeit better with volumetric density than with area density [24]. Other studies have shown positive associations [21] and good correlations between VBD and BI-RADS $[18,19]$. However, the use of correlation instead of agreement in previous studies makes direct comparison with this present study difficult. Furthermore, correlation may not be the method of choice since correlation only measures the strength of a relation between two variables, not the agreement between them $[32,33]$. However, there was a difference in mammographic density distribution according to BI-RADS between previous studies $[18,19]$ and this present study, which may be caused by differences in both age and ethnicity. Asian ethnicity and younger age are known to be associated with higher mammographic density $[13,34]$ as could be observed in the aforementioned studies. Gweon et al. reported $62 \%$ of the examinations to be categorized as BI-RADS 3 and $18.8 \%$ to be categorized as BI-RADS 4 in an Asian population with a mean age of 51.7 years [18]; the corresponding distribution for this study would be $35.2 \%$ for BI-RADS 3 and only $7.5 \%$ for BI-RADS 4 and a mean age of 58 years. The observations

\begin{tabular}{|c|c|c|c|}
\hline Radiologist & $\begin{array}{l}\text { Number of } \\
\text { women }\end{array}$ & & Kappa $(95 \%$ Cl) \\
\hline 1 & 208 & $\leftarrow$ & $0.66(0.56,0.75)$ \\
\hline 2 & 5068 & $\dot{\vdots}$ & $0.56(0.54,0.58)$ \\
\hline 3 & 1092 & - & $0.48(0.44,0.52)$ \\
\hline 4 & 993 & - & $0.52(0.48,0.56)$ \\
\hline 5 & 1065 & 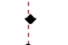 & $0.57(0.53,0.61)$ \\
\hline Overall & & 1 & $0.55(0.53,0.56)$ \\
\hline
\end{tabular}

Fig. 3 Agreement between Volpara density grade (VDG) and BI-RADS per radiologist
Table 2 Cross tabulation between BI-RADS scores and Volpara density grade (VDG)

\begin{tabular}{llllll}
\hline & \multicolumn{2}{l}{ BI-RADS $^{\mathrm{a}}$} & & & \\
\cline { 2 - 3 } VDG $^{\mathrm{b}}$ & 1 & 2 & 3 & 4 & Total \\
\hline 1 & 839 & 872 & 52 & 0 & 1763 \\
2 & 457 & 1730 & 513 & 8 & 2708 \\
3 & 76 & 788 & 1699 & 87 & 2650 \\
4 & 6 & 55 & 703 & 541 & 1305 \\
Total & 1378 & 3445 & 2967 & 636 & 8426 \\
\hline
\end{tabular}

${ }^{a}$ Breast Imaging Reporting and Data System

${ }^{\mathrm{b}}$ Volpara Density Grade

of this study, that Volpara classified more examinations in the highest VDG category than the radiologists (BI-RADS) and that there was moderate agreement between VDG and BIRADS, have also been previously described [18, 19, 22]. On the other hand, a previous Dutch study reported the BI-RADS distribution to be quite comparable with the VDG distribution, with a weighted kappa value of 0.80 [21].

There could be several explanations for the lower degree of agreement between Volpara and BI-RADS assessments. First, BI-RADS scores are set based on processed images, while Volpara analyses are performed on raw DM data. Second, VBD is measured on a continuous scale and BI-RADS scores are a coarse estimation into four groups. Therefore, values of mammographic density near the limits in the different VDG categories could be classified into the upper or the lower adjacent BI-RADS category since small differences in mammographic density would not be detected by the radiologists. And finally, both Volpara and the radiologists estimate the amount or percentage of dense breast tissue. However, despite the BIRADS 4th Edition definitions, it might be that the radiologists are also taking into account the distribution of the mammographic density and the difficulty of detecting a breast tumour, which may not always represent an actual increased amount of dense tissue, albeit a previous study reported high volumetric density to be correlated to decreased mammography sensitivity [35]. Taken together, this may indicate that radiologists evaluate mammographic density differently than automated software.

The automated method may still be a robust and valuable tool. High mammographic density, whether measured by Volpara or qualitatively with BI-RADS, has been shown to be associated with an increased breast cancer risk $[1,16,17]$. Previous reviews on mammographic density [2] and breast cancer risk prediction [3] have emphasized the need for improved and individualized breast cancer screening programs and risk prediction models. One way of improving these programs and models could be by incorporating a fully automated 
volumetric assessment of continuously measured mammographic density that may reduce the interobserver variability [15] and thereby producing a more reliable density estimate. A more reliable density estimate may then be used to stratify women in to different screening and risk groups.

Some limitations of this study require consideration. First, the BI-RADS 4th Edition was standard according to the time period during the main part of the MBTST; the impact of the BI-RADS 5th Edition on the results would have been interesting to analyze. This was, however, out of scope for this study. Second, two previous studies investigating BI-RADS agreement had several radiologists reading the images in the density analyses, which, of course, would have been preferable (11 [11] and 21 radiologists [12]). However, five radiologists is still a realistic number of readers in a single-centre study. Third, breast tumours are known to possibly affect the surrounding breast tissue and, thereby, perhaps also the mammographic density and we, therefore, excluded examinations from women with breast cancer. Finally, consistently registered information on previous breast surgery, use of hormone replacement therapy, or reproductive information was not available, all of which are factors known to possibly affect the mammographic density. However, we do not believe this affected our results because the aforementioned factors are not expected to affect the modes of assessment differently.

The population in this study was a screening population representative of the female population in the screening ages 40-74 years in the city of Malmö, Sweden [27]. Furthermore, the BI-RADS scores were prospectively performed by several radiologists, representing the common mass screening setting. The interobserver variability was low, reflecting a solid evaluation of qualitatively estimated mammographic density. Altogether, this study may well represent everyday screening practice.

In conclusion, there was moderate agreement between Volpara and BI-RADS scores from European radiologists, indicating that radiologists evaluate mammographic density differently than automated software. However, the automated method may still be a robust and valuable tool. In addition to this, the differences in interpretation between radiologists and software will require further investigation. Future studies evaluating fully automated density assessments on different populations are warranted in order to ensure accurate reflection of mammographic density, with an additional focus on breast cancer risk and screening outcomes.

\footnotetext{
Acknowledgements Ralph Highnam at Volpara is acknowledged for providing access to the Volpara software. The radiologists participating in the BI-RADS scores: Ingvar Andersson, Annicka Lindahl, Marianne Löfgren, Cecilia Wattsgård and Barbara Ziemiecka, and the nurses responsible for performing the screening examinations: Ulrica Pettersson and Maria Seserin.

The scientific guarantor of this publication is Pontus Timberg, $\mathrm{PhD}$. The authors of this manuscript declare relationships with the following
}

companies: The sponsors (Volpara) of the study had no role in the design and performance of the study, data analysis, or data interpretation. Siemens AG (Erlangen, Germany) sponsored the study by providing the mammography equipment. KL, SZ, and PT have received speakers' fees and travel grants from Siemens. This work has received funding from government funding for clinical research within the National Health Services, Research Foundation, and The Swedish Cancer Society. One of the authors (AR) has significant statistical expertise. Institutional Review Board approval was obtained. Written informed consent was obtained from all subjects (patients) in this study. Some study subjects or cohorts have been previously reported in:

Lang et al.: "Performance of one-view breast tomosynthesis as a standalone breast cancer screening modality: results from the Malmo Breast Tomosynthesis Screening Trial, a population-based study". European Radiology 2016, 26(1):184-190. Lang et al.: "False positives in breast cancer screening with one-view breast tomosynthesis: an analysis of findings leading to recall, work-up and biopsy rates in the Malmö Breast Tomosynthesis Screening Trial" (accepted in European Radiology 2015). Rosso et al.: "Factors affecting recall rate and false positive fraction in breast cancer screening with breast tomosynthesis - A statistical approach". The Breast 2015, 24(5):680-686.

Methodology: prospective, diagnostic or prognostic study, performed at one institution.

Open Access This article is distributed under the terms of the Creative Commons Attribution 4.0 International License (http:// creativecommons.org/licenses/by/4.0/), which permits unrestricted use, distribution, and reproduction in any medium, provided you give appropriate credit to the original author(s) and the source, provide a link to the Creative Commons license, and indicate if changes were made.

\section{References}

1. McCormack VA, dos Santos SI (2006) Breast density and parenchymal patterns as markers of breast cancer risk: a meta-analysis. Cancer Epidemiol Biomarkers Prev 15:1159-1169

2. Huo CW, Chew GL, Britt KL et al (2014) Mammographic density-a review on the current understanding of its association with breast cancer. Breast Cancer Res Treat 144:479-502

3. Howell A, Anderson AS, Clarke RB et al (2014) Risk determination and prevention of breast cancer. Breast Cancer Res 16:446

4. Yaffe MJ (2008) Mammographic density. Measurement of mammographic density. Breast Cancer Res 10:209

5. D'Orsi CJSE, Mendelson EB, Morris EA et al (2013) ACR BIRADS ${ }^{\circledR}$ Atlas, breast imaging reporting and data system. American College of Radiology, Reston

6. Berg WA, Campassi C, Langenberg P, Sexton MJ (2000) Breast imaging reporting and data system: inter- and intraobserver variability in feature analysis and final assessment. AJR Am J Roentgenol 174:1769-1777

7. Ciatto S, Houssami N, Apruzzese A et al (2005) Categorizing breast mammographic density: intra- and interobserver reproducibility of BI-RADS density categories. Breast 14:269-275

8. Kerlikowske K, Grady D, Barclay J et al (1998) Variability and accuracy in mammographic interpretation using the American College of Radiology Breast Imaging Reporting and Data System. J Natl Cancer Inst 90:1801-1809

9. Nicholson BT, LoRusso AP, Smolkin M, Bovbjerg VE, Petroni GR, Harvey JA (2006) Accuracy of assigned BI-RADS breast density category definitions. Acad Radiol 13:1143-1149

10. Ooms EA, Zonderland HM, Eijkemans MJC et al (2007) Mammography: interobserver variability in breast density assessment. Breast 16:568-576 
11. Ciatto S, Bernardi D, Calabrese $M$ et al (2012) A first evaluation of breast radiological density assessment by QUANTRA software as compared to visual classification. Breast 21:503-506

12. Sacchetto D, Morra L, Agliozzo S et al (2016) Mammographic density: comparison of visual assessment with fully automatic calculation on a multivendor dataset. Eur Radiol 26:175-183

13. Boyd NF, Martin LJ, Yaffe MJ, Minkin S (2011) Mammographic density and breast cancer risk: current understanding and future prospects. Breast Cancer Res 13:223

14. Shepherd JA, Kerlikowske K, Ma L et al (2011) Volume of mammographic density and risk of breast cancer. Cancer Epidemiol Biomarkers Prev 20:1473-1482

15. Alonzo-Proulx O, Mawdsley GE, Patrie JT, Yaffe MJ, Harvey JA (2015) Reliability of automated breast density measurements. Radiology 275:366-376

16. Park IH, Ko K, Joo J et al (2014) High volumetric breast density predicts risk for breast cancer in postmenopausal, but not premenopausal, Korean women. Ann Surg Oncol 21:4124-4132

17. Eng A, Gallant Z, Shepherd J et al (2014) Digital mammographic density and breast cancer risk: a case inverted question mark control study of six alternative density assessment methods. Breast Cancer Res 16:439

18. Gweon HM, Youk JH, Kim JA, Son EJ (2013) Radiologist assessment of breast density by BI-RADS categories versus fully automated volumetric assessment. AJR Am J Roentgenol 201:692-697

19. Seo JM, Ko ES, Han BK, Ko EY, Shin JH, Hahn SY (2013) Automated volumetric breast density estimation: a comparison with visual assessment. Clin Radiol 68:690-695

20. Lee HN, Sohn YM, Han KH (2014) Comparison of mammographic density estimation by Volpara software with radiologists' visual assessment: analysis of clinical-radiologic factors affecting discrepancy between them. Acta Radiol 56:1061-1068

21. van der Waal D, den Heeten GJ, Pijnappel RM et al (2015) Comparing visually assessed BI-RADS breast density and automated volumetric breast density software: a cross-sectional study in a breast cancer screening setting. PLoS One 10, e0136667

22. Gubern-Merida A, Kallenberg M, Platel B, Mann RM, Marti R, Karssemeijer N (2014) Volumetric breast density estimation from full-field digital mammograms: a validation study. PLoS One 9, e85952

23. Wang J, Azziz A, Fan B et al (2013) Agreement of mammographic measures of volumetric breast density to MRI. PLoS One 8, e81653

24. Morrish OW, Tucker L, Black R, Willsher P, Duffy SW, Gilbert FJ (2015) Mammographic breast density: comparison of methods for quantitative evaluation. Radiology 275:356-365

25. Sandberg ME, Li J, Hall P et al (2013) Change of mammographic density predicts the risk of contralateral breast cancer-a case-control study. Breast Cancer Res 15:R57

26. Cuzick J, Warwick J, Pinney E et al (2011) Tamoxifen-induced reduction in mammographic density and breast cancer risk reduction: a nested case-control study. J Natl Cancer Inst 103:744-752

27. Lang K, Andersson I, Rosso A, Tingberg A, Timberg P, Zackrisson S (2015) Performance of one-view breast tomosynthesis as a standalone breast cancer screening modality: results from the Malmo Breast Tomosynthesis Screening Trial, a population-based study. Eur Radiol 26:184-190

28. EUREF. Available via www.EUREF.org

29. Highnam R, Brady S, Yaffe M, Karssemeijer N, Harvey J (2010) Robust breast composition measurement - VolparaTM. In: Martí J, Oliver A, Freixenet J, Martí R (eds) Digital mammography. Springer, Berlin, pp 342-349 (Lecture Notes in Computer Science)

30. Sun S (2011) Meta-analysis of Cohen's Kappa. Health Serv Outcome Res Methodol 11:145-163

31. McHugh ML (2012) Interrater reliability: the kappa statistic. Biochem Med (Zagreb) 22:276-282

32. Martin Bland J, Altman D (1986) Statistical methods for assessing agreement between two methods of clinical measurement. Lancet 327:307-310

33. Rosso A (2015) Correlation does not mean agreement: why is it still used as a synonym of agreement? Radiology 276:617-619

34. Maskarinec G, Meng L, Ursin G (2001) Ethnic differences in mammographic densities. Int J Epidemiol 30:959-965

35. Wanders JO, Holland K, Veldhuis WB, Mann RM, Peeters PH, van Gils CH, Karssemeijer N (2015) Effect of volumetric mammographic density on performance of a breast cancer screening program using full-field digital mammography. European Congress of Radiology, Vienna 